\title{
Synthesis of a C-glycoside analogue of $\beta$-D-galactosyl
}

\section{hydroxylysine and incorporation in a glycopeptide from type II}

\section{collagen.}

\author{
Tomas Gustafsson, ${ }^{a}$ Mattias Hedenström, ${ }^{a}$ and Jan Kihlberg*a, b \\ ${ }^{a}$ Organic Chemistry, Department of Chemistry, Umeå University, SE-901 87 Umeå, \\ Sweden and ${ }^{\mathrm{b}}$ AstraZeneca R\&D Mölndal, SE-431 83 Mölndal, Sweden.

\section{jan.kihlberg@chem.umu.se}

S2: General Methods and Materials

S3: $\quad{ }^{1} \mathrm{H}$ NMR for compound 4

S4: $\quad{ }^{13} \mathrm{C}$ NMR for compound 4

S5: $\quad{ }^{1} \mathrm{H}$ NMR for compound 5

S6: $\quad{ }^{13} \mathrm{C}$ NMR for compound 5

S7: $\quad{ }^{1} \mathrm{H}$ NMR for compound 7

S8: $\quad{ }^{13} \mathrm{C}$ NMR for compound 7

S9: $\quad{ }^{1} \mathrm{H}$ NMR for compound 8

S10: $\quad{ }^{13} \mathrm{C}$ NMR for compound 8

S11: ${ }^{1} \mathrm{H}$ NMR for compound 9

S12: $\quad{ }^{13} \mathrm{C}$ NMR for compound 9

S13: Assignment for compound 11

S14: ${ }^{1} \mathrm{H}$ NMR for compound $\mathbf{1 1}$ (2 isomers)

S15: $\quad{ }^{1} \mathrm{H}$ NMR for compound 11 (2 isomers)

S16 ${ }^{1} \mathrm{H}$ NMR for compound $\mathbf{1 2}$

S17: $\quad{ }^{13} \mathrm{C}$ NMR for compound 12

S18: ${ }^{1} \mathrm{H}$ NMR for compound 13

S19 $\quad{ }^{13} \mathrm{C}$ NMR for compound 13

S20: ${ }^{1} \mathrm{H}$ NMR for compound $\mathbf{1 4}$

S21: $\quad{ }^{13}$ C NMR for compound 14

S22 ${ }^{1} \mathrm{H}$ NMR for compound 15

S23: $\quad{ }^{13} \mathrm{C}$ NMR for compound 15

S24: $\quad{ }^{1} \mathrm{H}$ NMR for compound $\mathbf{1 6}$

S25 ${ }^{13} \mathrm{C}$ NMR for compound 16

S26: ${ }^{1} \mathrm{H}$ NMR for compound 1

S27: $\quad{ }^{13} \mathrm{C}$ NMR for compound 1 
General Methods and Materials. All reactions were carried out under an inert atmosphere with dry solvents under anhydrous conditions, unless otherwise stated. $\mathrm{CH}_{2} \mathrm{Cl}_{2}$ was distilled from calcium hydride, whereas THF and toluene were distilled from potassium benzophenone and sodium, respectively. Methanol was dried over $3 \AA$ molecular sieves. TLC was performed on Silica gel 60 F254 with detection by UV light and staining with a solution of ethanolic phosphomolybdic acid. Flash column chromatography (eluents given in brackets) was performed on silica gel. Microwave reactions were performed in a monomode reactor using 2-5 $\mathrm{mL}$ roundbottom process vials sealed with teflon septa and aluminum crimp tops. Temperature measurments were performed by infrared detection and reaction times are given as total irradiation time.

${ }^{1} \mathrm{H}$ and ${ }^{13} \mathrm{C}$ NMR spectra for compounds 4-15 were recorded at 400 and 100 $\mathrm{MHz}$, respectively, for solutions in $\mathrm{CDCl}_{3}$ [residual $\mathrm{CHCl}_{3}\left(\delta_{\mathrm{H}} 7.26 \mathrm{ppm}\right)$ or $\mathrm{CDCl}_{3}\left(\delta_{\mathrm{C}}\right.$ $77.0 \mathrm{ppm}$ ), as internal standard] at $298 \mathrm{~K} .{ }^{1} \mathrm{H}$ NMR and ${ }^{13} \mathrm{C}$ NMR spectra for compound 1 were recorded at 400 and $100 \mathrm{MHz}$, respectively, for solutions in $\mathrm{MeOH}-\mathrm{d}_{4}$ [residual $\mathrm{MeOH}-\mathrm{d}_{3}\left(\delta_{\mathrm{H}} 3.30 \mathrm{ppm}\right)$ or $\mathrm{MeOH}-\mathrm{d}_{4}\left(\delta_{\mathrm{C}} 49.9 \mathrm{ppm}\right)$ as internal standard] at $298 \mathrm{~K}$. Peptide spectra were recorded at $500 \mathrm{MHz}$ for solutions in $\mathrm{H}_{2} \mathrm{O}\left(10 \% \mathrm{D}_{2} \mathrm{O}\right)\left[\mathrm{H}_{2} \mathrm{O}\left(\delta_{\mathrm{H}}\right.\right.$ $4.98 \mathrm{ppm}$ ) as internal standard]. First-order chemical shifts and coupling constants were obtained from onedimensional spectra; carbon and proton resonances were assigned from COSY and HETCOR experiments. Optical rotations were measured at $20{ }^{\circ} \mathrm{C}$. Determinations of diastereomeric excesses were performed with HPLC on a staight-phase column (Chiracel OD, Heptane-EtOH 97:3 $1 \mathrm{~mL} / \mathrm{min}$ flow) and after establishing a good correlation with ${ }^{1} \mathrm{H}$ NMR, by using ${ }^{1} \mathrm{H}$ NMR spectroscopy. 
总

69.9ट

Oे

$\ddot{0}$

$\circ-28 \div-$

$66^{\circ} t$

$\angle 0^{\circ} \mathrm{Z}$

$-\int \begin{aligned} & 00^{\circ} \tau \\ & 26^{\circ} 0 \\ & \\ & 60^{\circ} \tau \\ & 86^{\circ} 0\end{aligned}$

86.0

$-\underbrace{}_{0 .}$

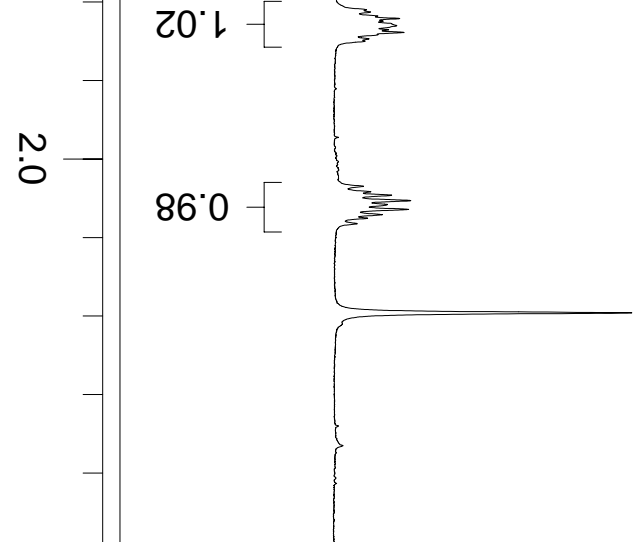




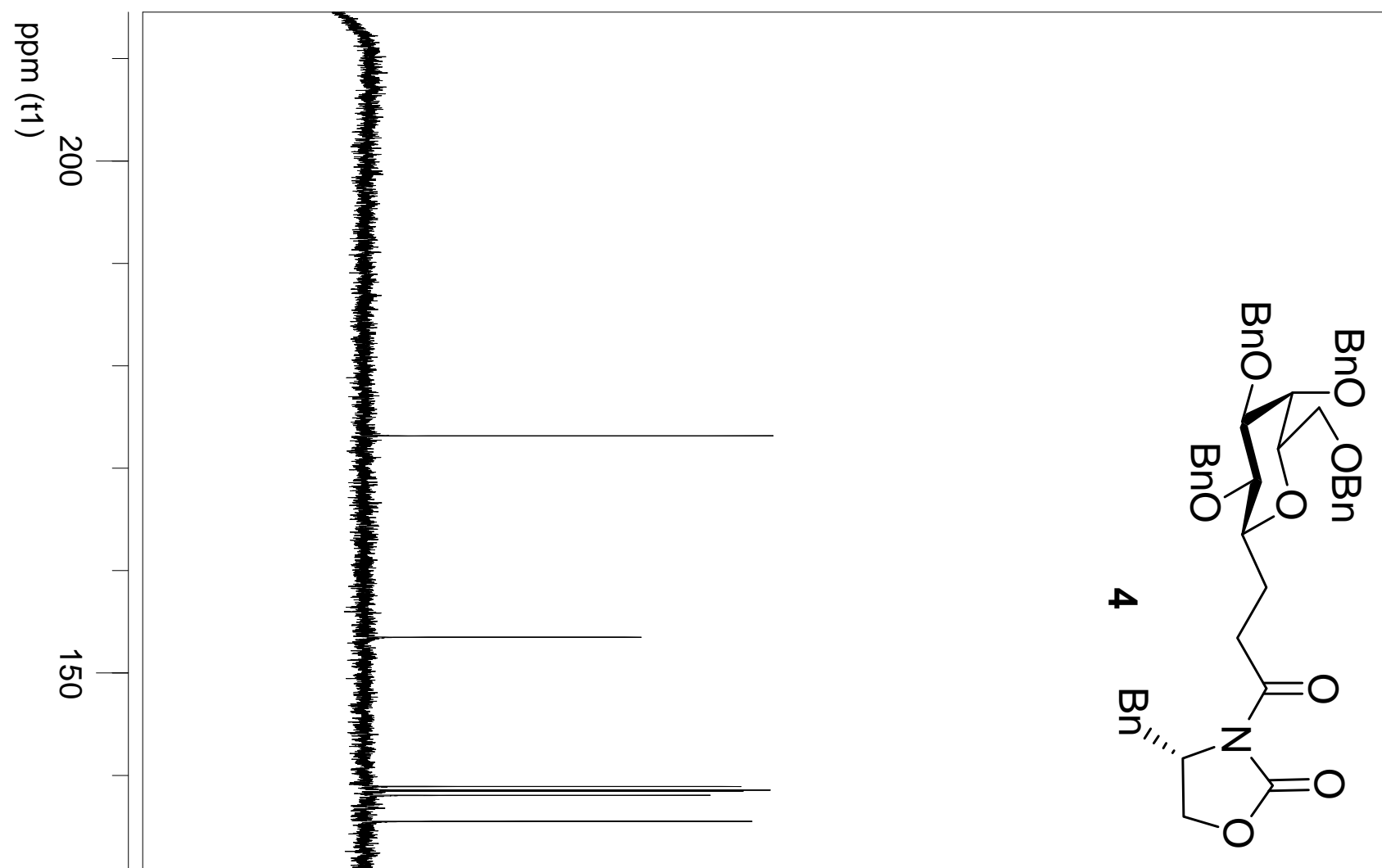

$\vec{a}$

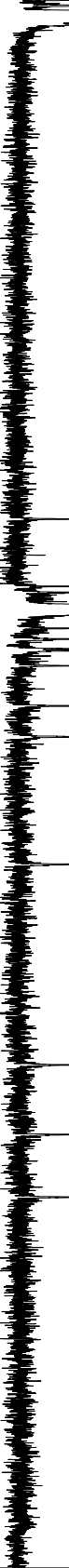


$\frac{0}{0}$
3

$6 t^{\circ} 92$

ò

$\stackrel{\circ}{\circ}-106.0$

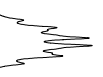

$G$

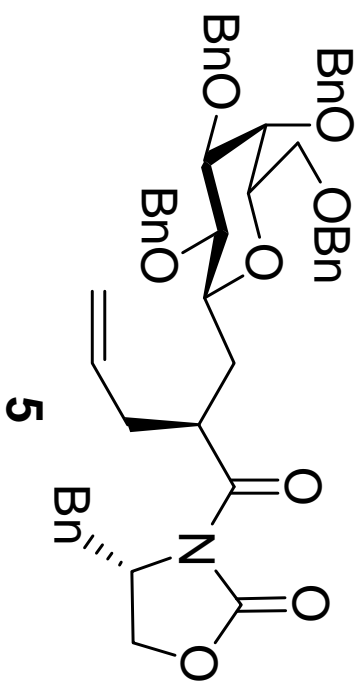

$16^{\circ} \varepsilon$

0

$-20 \%$

$10^{\circ} \mathrm{L}$

$00^{\circ}$.

+0
$\circ-1$
$86 \%$

$-766^{\circ}$

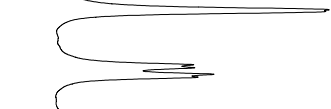

$\omega$

$66^{\circ} \mathrm{L}$
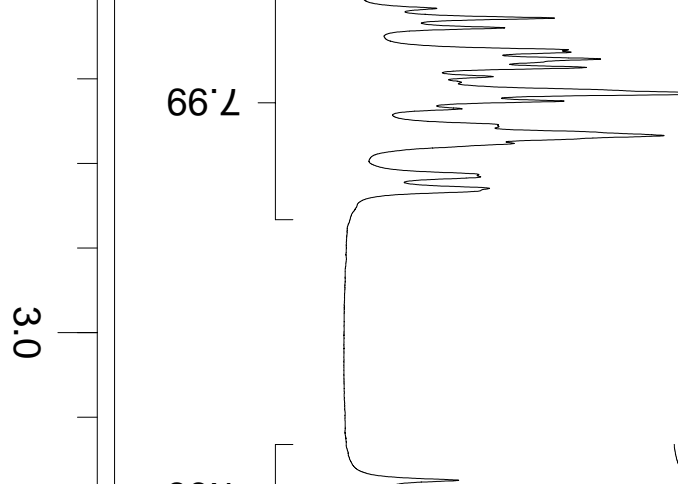

N

$96 \%$

$70 \%$

$00 \%$

$\varepsilon 0$.

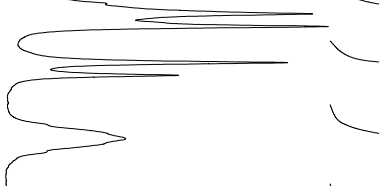




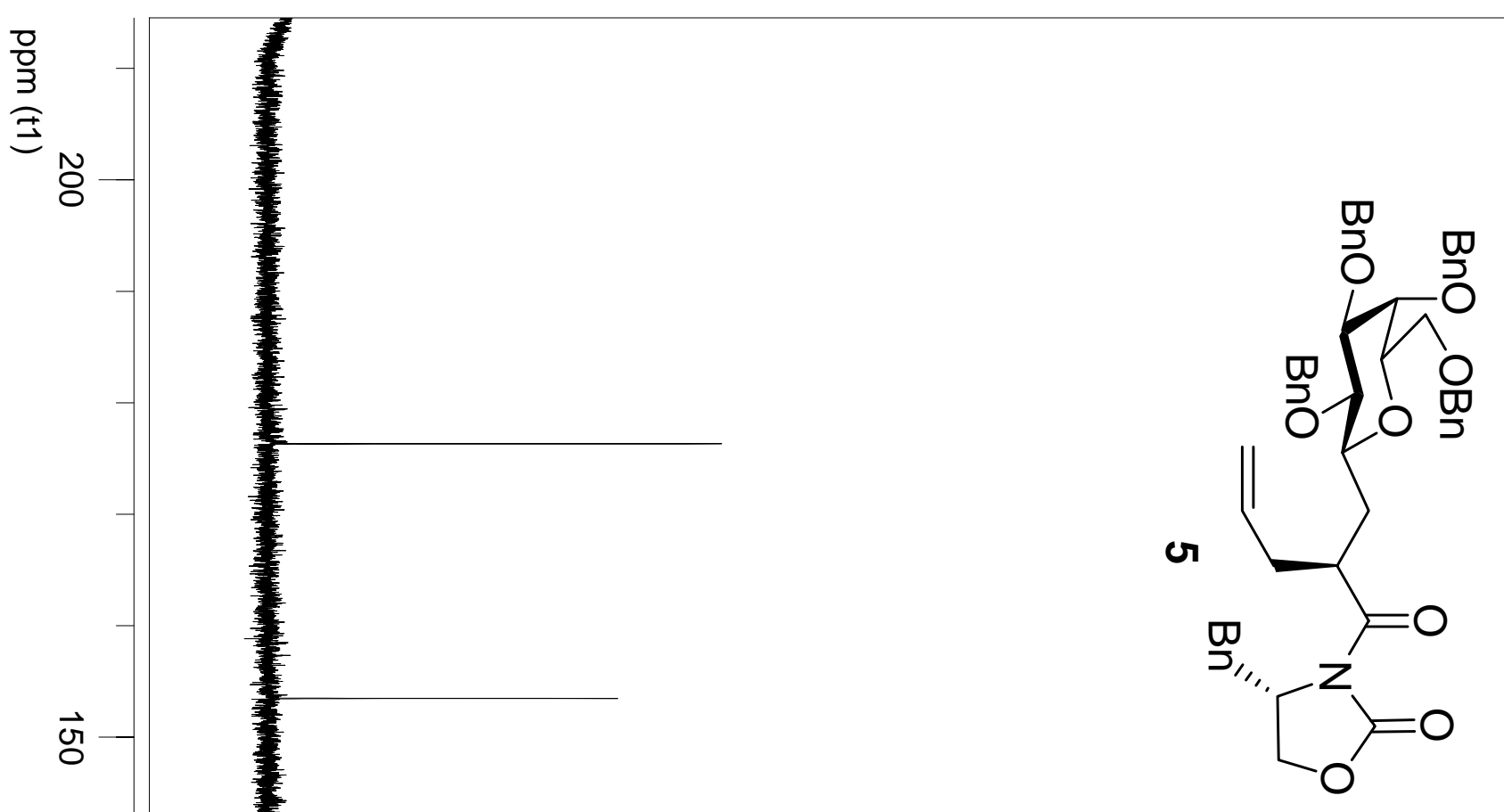

$\overrightarrow{8}$

잉 


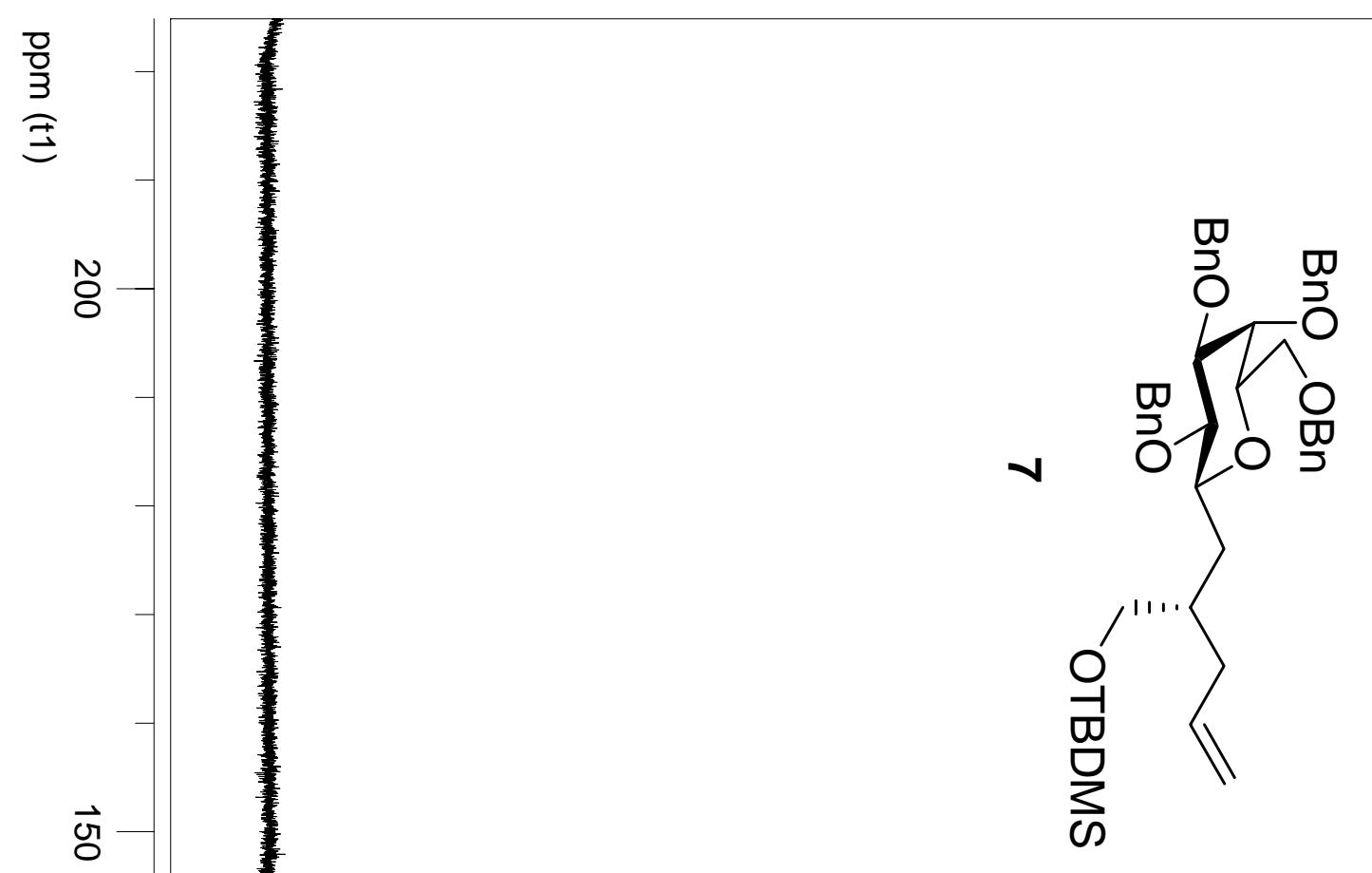

$\vec{\circ}$

o 


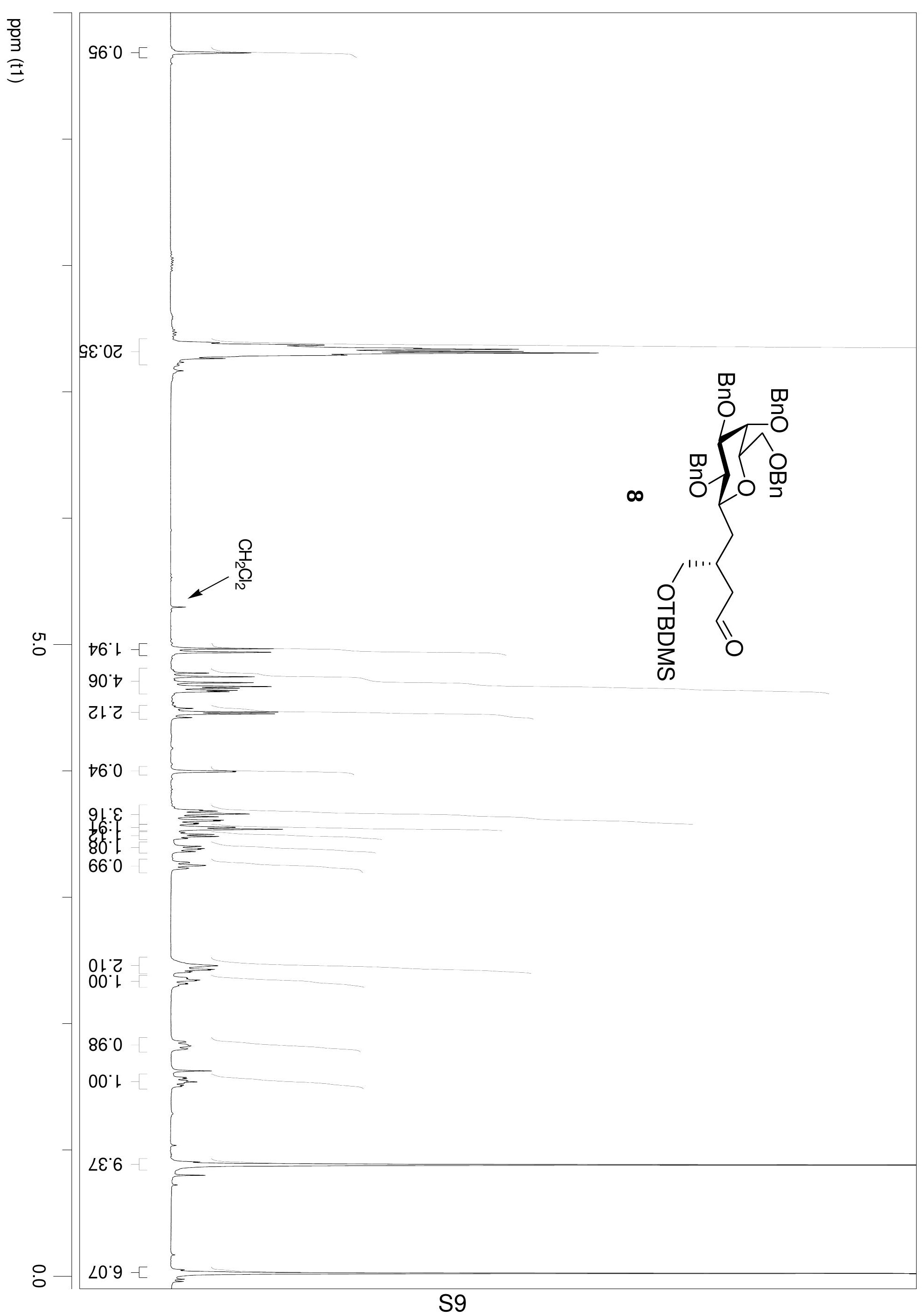



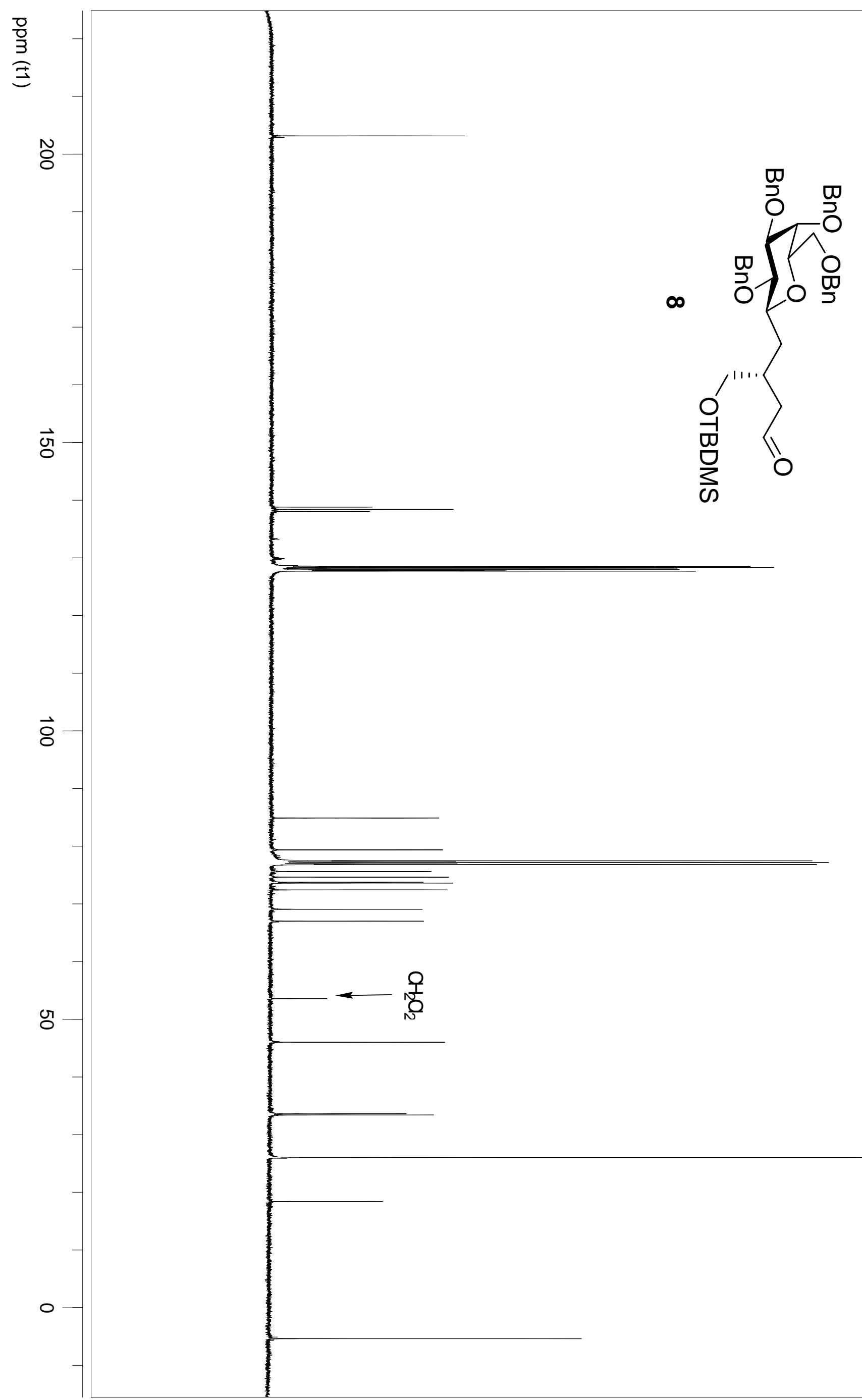
$\frac{0}{3}$
3

2082

ㄱ- $18.0-$

$\angle 6^{\circ} 0$

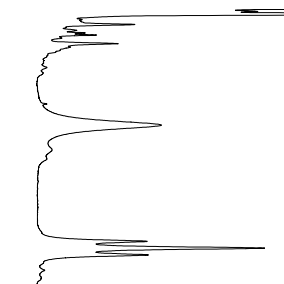

0

$12 \cdot 2$

0

102

ำเ

9เ乙-

$\ddot{0}$

$\ddot{0}$

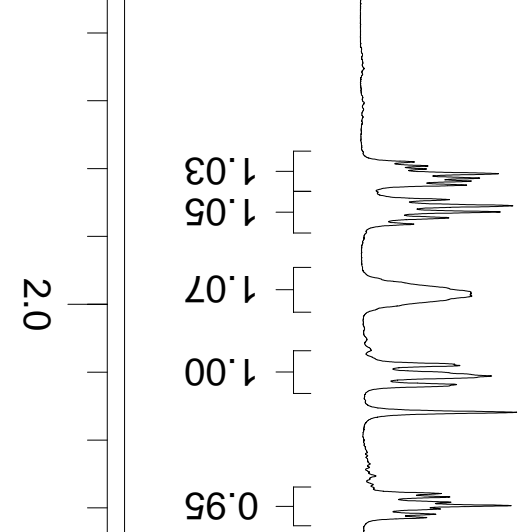

10.1

$\varepsilon 6^{\circ} \mathrm{C}$

ट†"†

$0 S^{\circ} \mathrm{C}$

$\angle 0^{\circ} \mathrm{C}$

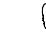
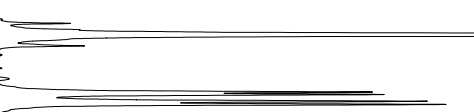

6

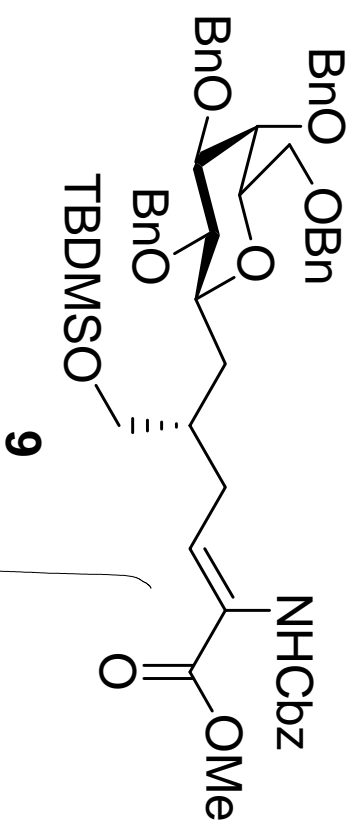

0
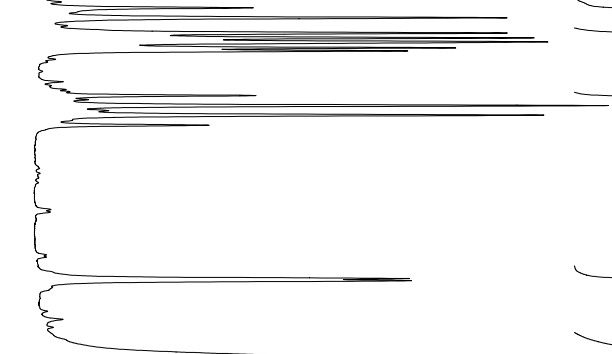

(1)
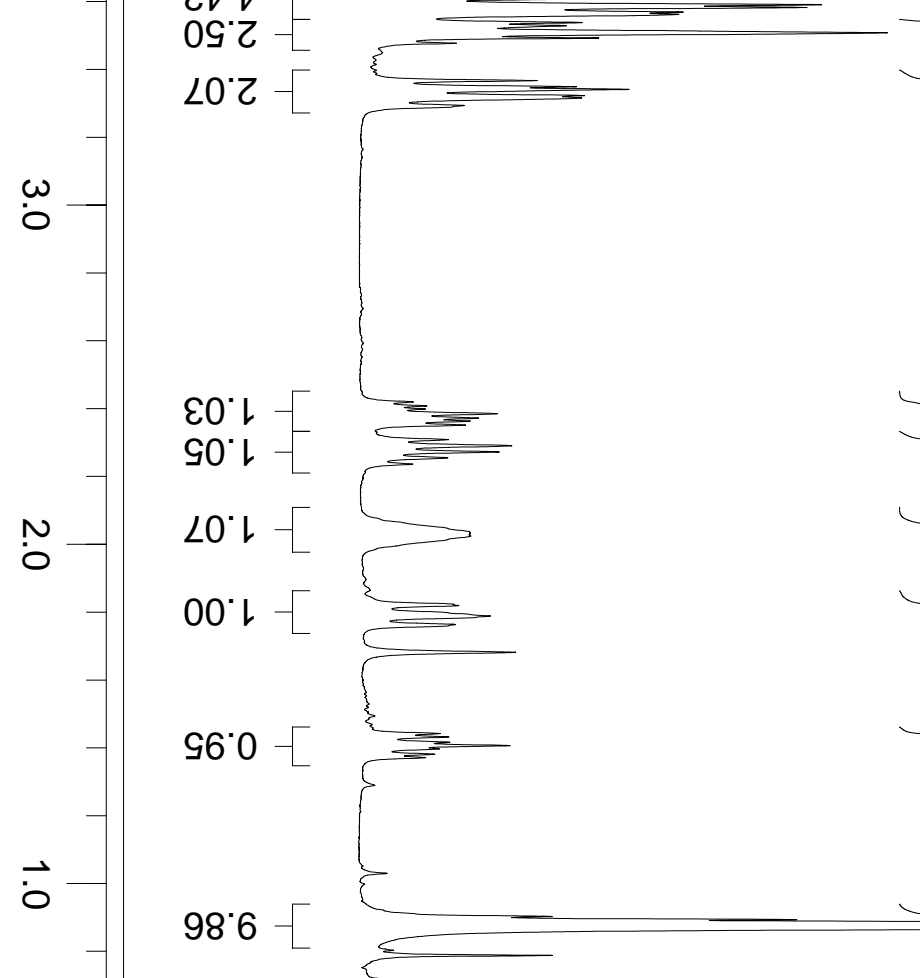

: $97^{\circ} 9-[$ 


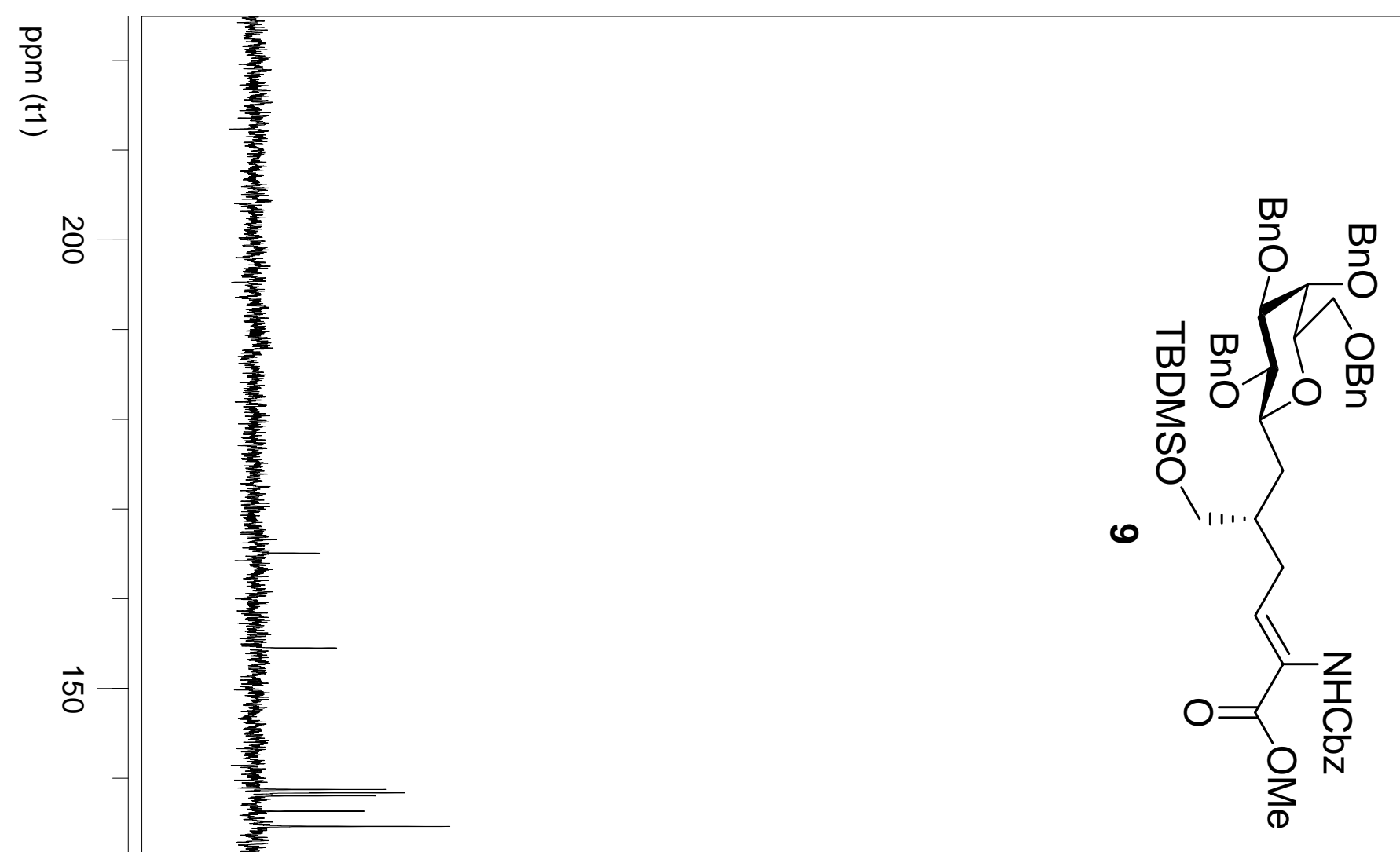

-

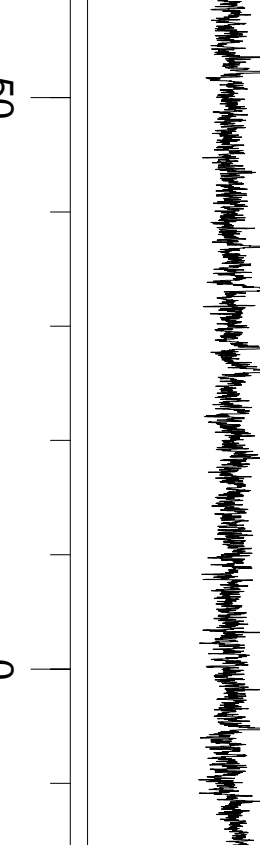

오ำ

佥 
Assignment for:

Benzyloxycarbonylamino-[(4S)-(2,3,4,6-tetra-O-benzyl- $\beta$-D-

galactopyranosylmethyl)-tetrahydro-furan-2-yl]-acetic acid methyl ester 10

Fraction 1, two isomers, 10a: ${ }^{1} \mathrm{H} \mathrm{NMR}\left(\mathrm{CDCl}_{3}, 400 \mathrm{MHz}\right): \delta=7.39-7.23(\mathrm{~m}, 25 \mathrm{H}, \mathrm{Ph})$, 5.45 and $5.41(2 \mathrm{~d}, 1 \mathrm{H}, J=9.5$ and $8.8 \mathrm{~Hz}, \mathrm{NH}), 5.15-5.08\left(\mathrm{~m}, 2 \mathrm{H}, \mathrm{NCOOCH}_{2}\right), 4.97-$ $4.88\left(\mathrm{~m}, 2 \mathrm{H}, \mathrm{PhCH}_{2} \mathrm{O}\right), 4.77-4.58\left(\mathrm{~m}, 4 \mathrm{H}, \mathrm{PhCH}_{2} \mathrm{O}\right), 4.46-4.34\left(\mathrm{~m}, 3 \mathrm{H}, \mathrm{PhCH}_{2} \mathrm{O}, \mathrm{H}-\alpha\right)$, 4.30-4.26 and 4.18-4.10 (2m, 1H, H- $\beta), 4.04-3.93(\mathrm{~m}, 2 \mathrm{H}, \mathrm{H}-4, \mathrm{H}-\varepsilon), 3.75$ and 3.69 (2s, $3 \mathrm{H}, \mathrm{OCH}_{3}$ ), 3.65-3.52 (m, 2H, H-2, H-3), 3.52-3.44 (m, 3H, H-5, H-6), 3.30 (t, 1H, J= $8.3 \mathrm{~Hz}, \mathrm{H}-\varepsilon), 3.17$ (t, 1H, J = 9.0 Hz, H-1), 2.35-2.24 (m, 1H, H- $), 1.97-1.82$ (m, 2H, H$\left.\gamma, \mathrm{CH}_{2}\right), 1.72-1.58(\mathrm{~m}, 1 \mathrm{H}, \mathrm{H}-\gamma), 1.50-1.37\left(\mathrm{~m}, 1 \mathrm{H}, \mathrm{CH}_{2}\right)$.

Fraction 2, two isomers, 10b: ${ }^{1} \mathrm{H} \mathrm{NMR}\left(\mathrm{CDCl}_{3}, 400 \mathrm{MHz}\right): \delta=7.40-7.21(\mathrm{~m}, 25 \mathrm{H}, \mathrm{Ph})$, 5.52 and $5.42(2 \mathrm{~d}, 1 \mathrm{H}, J=9.4$ and $8.5 \mathrm{~Hz}, \mathrm{NH}), 5.19-5.05\left(\mathrm{~m}, 2 \mathrm{H}, \mathrm{NCOOCH}_{2}\right), 4.97-$ 4.88 (m, 2H, $\left.\mathrm{PhCH}_{2} \mathrm{O}\right), 4.76-4.59$ (m, 4H, $\left.\mathrm{PhCH}_{2} \mathrm{O}\right), 4.51-4.35$ (m, 3H, $\left.\mathrm{PhCH}_{2} \mathrm{O}, \mathrm{H}-\alpha\right)$, 4.28-4.23 and 4.18-4.10 (2m, $1 \mathrm{H}, \mathrm{H}-\beta), 4.00-3.87(\mathrm{~m}, 2 \mathrm{H}, \mathrm{H}-4, \mathrm{H}-\varepsilon), 3.75$ and $3.70(2 \mathrm{~s}$, $\left.3 \mathrm{H}, \mathrm{OCH}_{3}\right), 3.65-3.51$ (m, 2H, H-2, H-3), 3.50-3.44 (m, 3H, H-5, H-6), 3.41-3.28 (m, 1H,

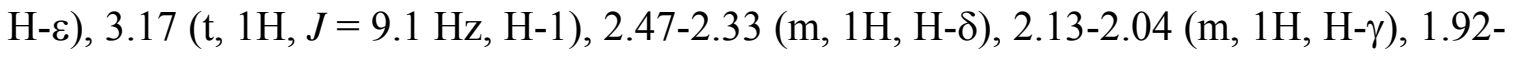
$1.83\left(\mathrm{~m}, 1 \mathrm{H}, \mathrm{CH}_{2}\right), \quad 1.54-1.45(\mathrm{~m}, 1 \mathrm{H}, \mathrm{H}-\gamma), 1.45-1.34\left(\mathrm{~m}, 1 \mathrm{H}, \mathrm{CH}_{2}\right)$. 


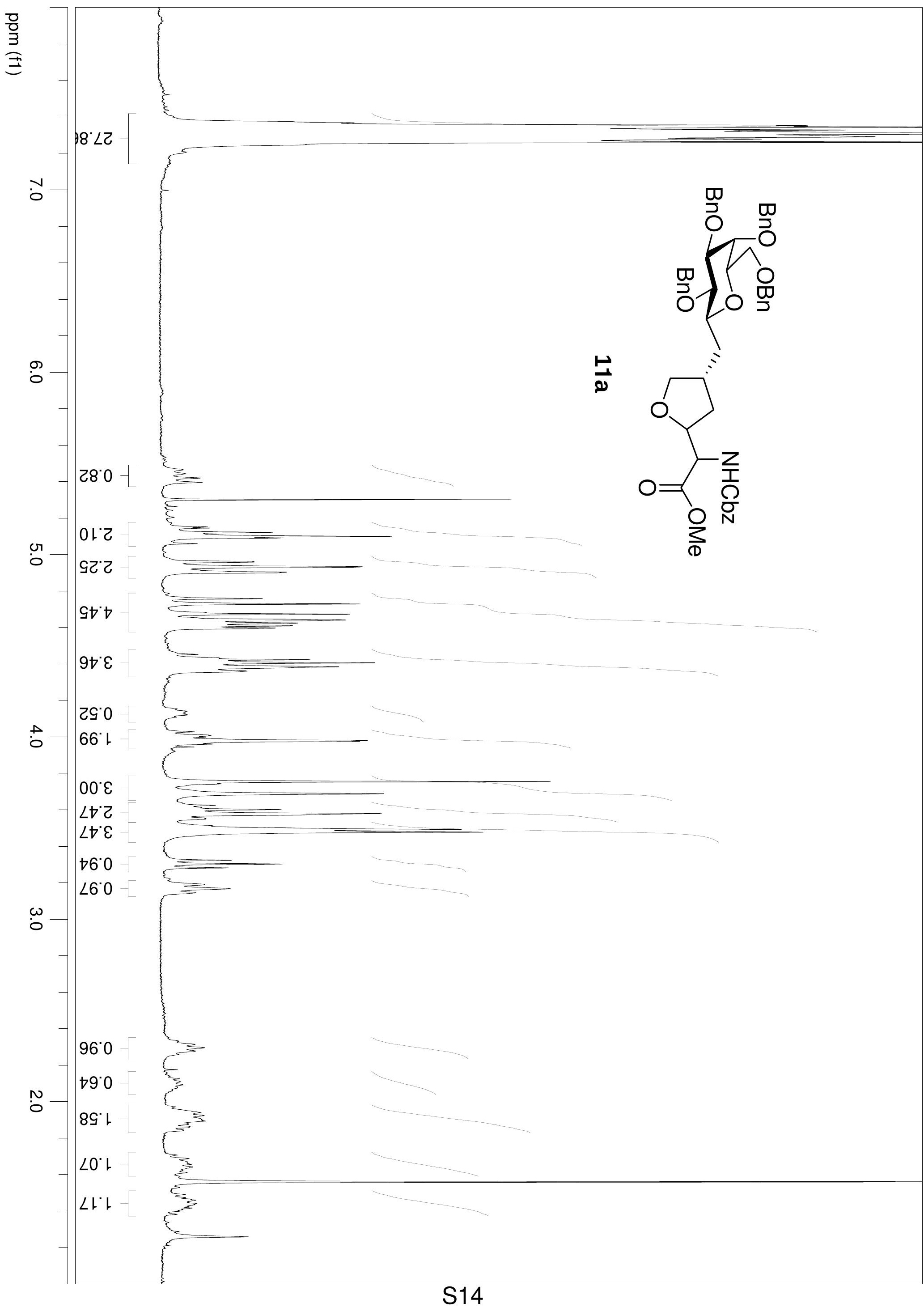


$\frac{0}{0}$
3
플

99.82

O)

0

0

627

Sट'

$89^{\circ} \mathrm{t}$

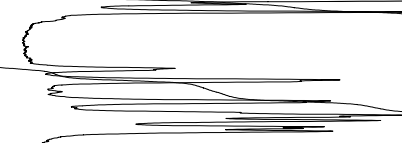

$-\varepsilon 6^{\circ} \varepsilon$

$89^{\circ} 0$
$\angle 8$.

$66^{\circ} 2$

$S L ' Z$

$\angle 8^{\circ} \mathcal{E}$

$\mathrm{SZ}$

$\omega$

00 เ
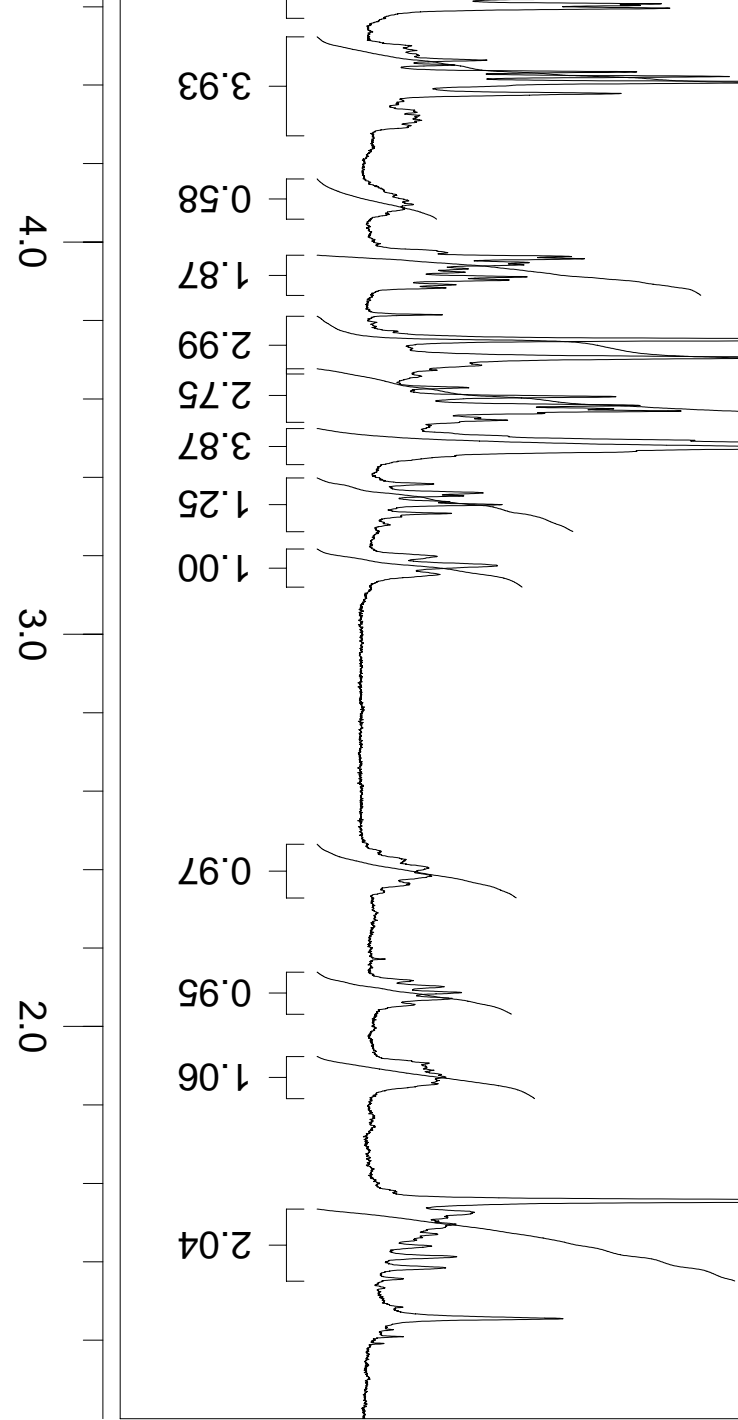
$\frac{0}{0}$
3
ㅍ

$\stackrel{8}{8}-960-$

Oे

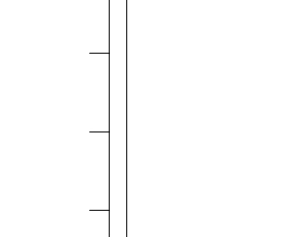

$0-90^{\circ} 2-[$

$-\begin{aligned} & 961- \\ & 90 \% \\ & 108\end{aligned}$

$-\begin{aligned} & 10^{\circ} \varepsilon \\ & 00^{\circ} z\end{aligned}$

$\varepsilon 0.1-[$

$+56^{\circ} 0=$

$-1$
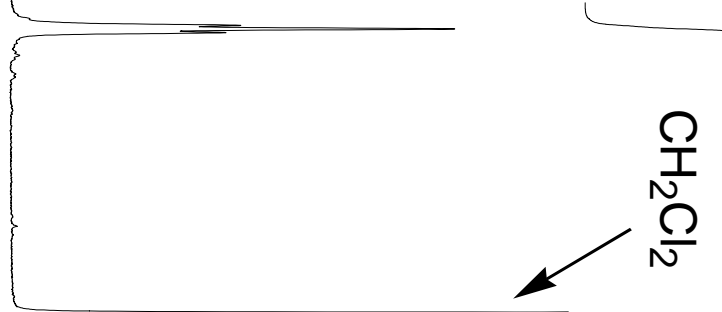

$\frac{\frac{0}{1}}{\frac{0}{0}}$

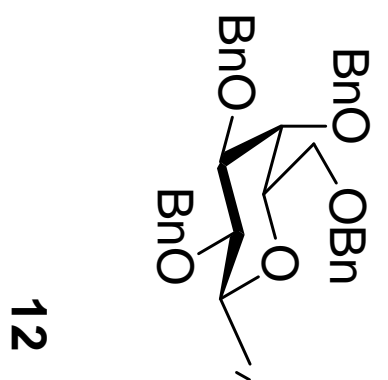

$-98^{\circ} L-$

$\omega$

- 760

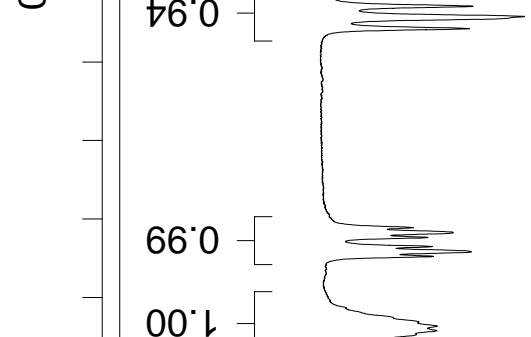

$\stackrel{0}{\circ}$

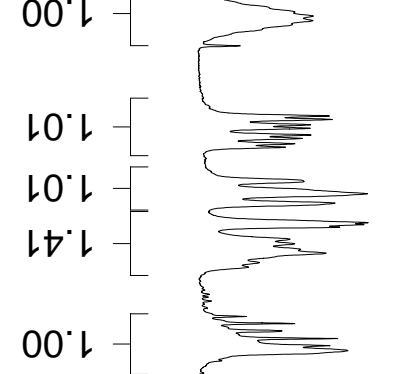


응

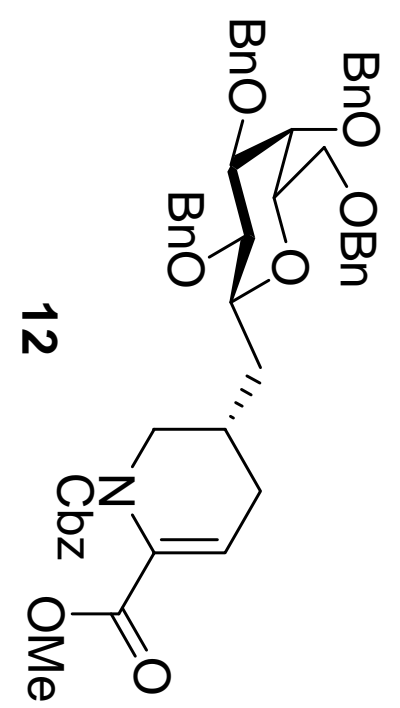

ज्ञ

잉

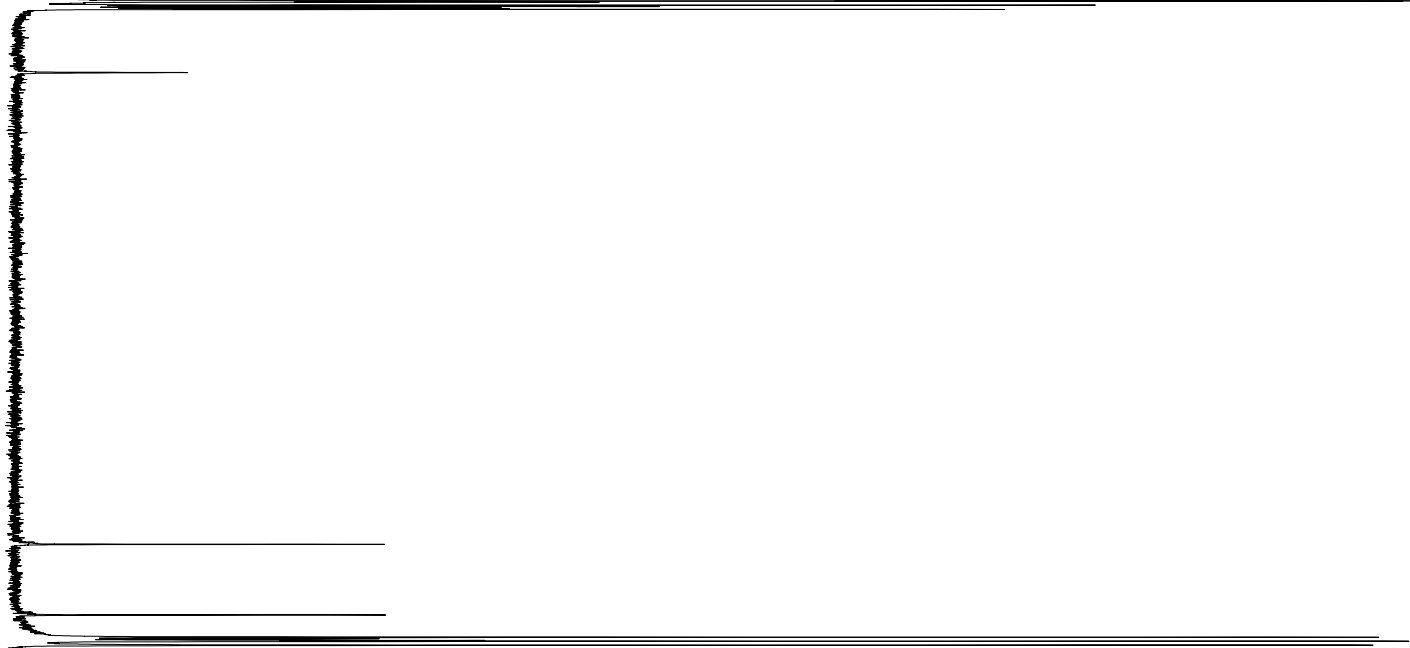

0

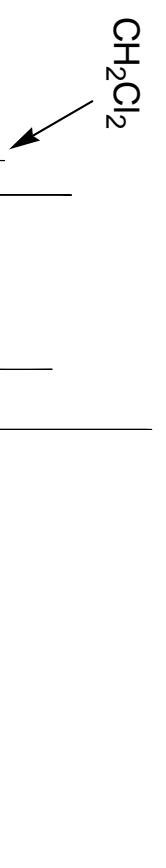


$\frac{0}{3}$
3

$67 \cdot 92-$

v

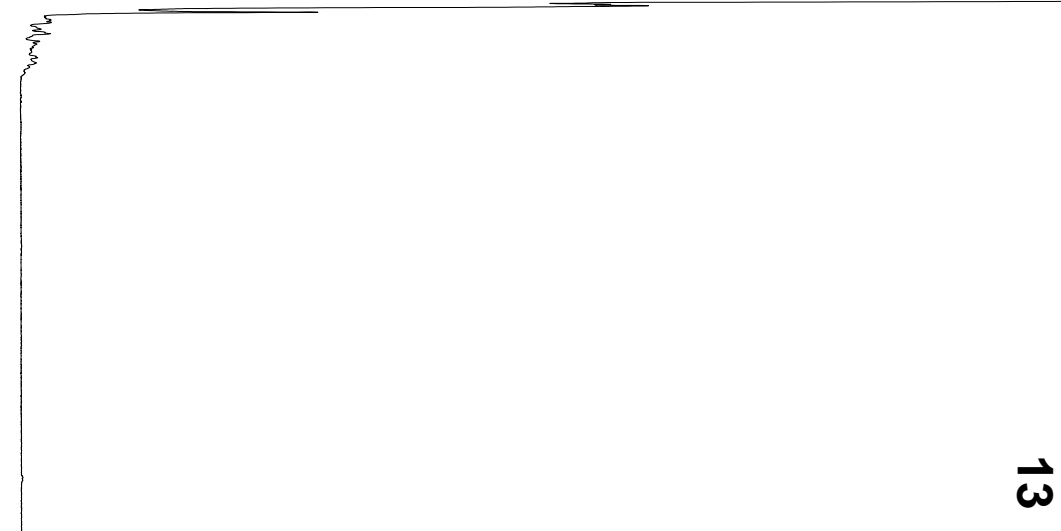

0

$\vec{\omega}$

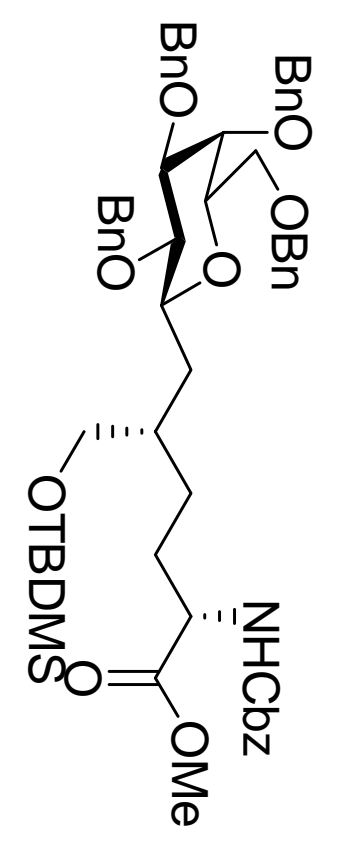

ต้.'

乙レ'こ -

$68^{\circ} 0$

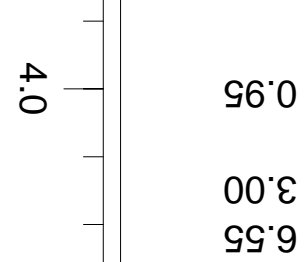

90. -

$00^{\circ}$.

$\omega$
0

N

0

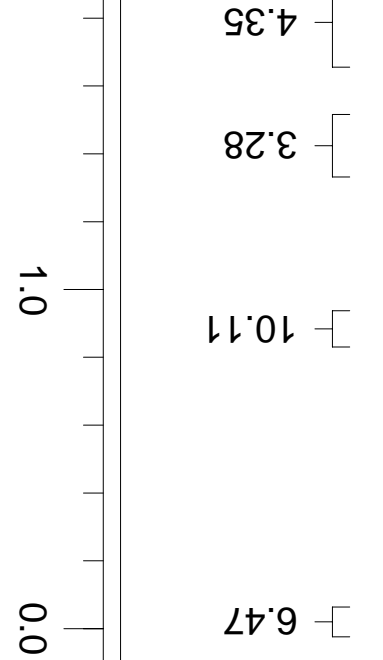


응
플

$\angle 0.82-$

$\stackrel{v}{0}$
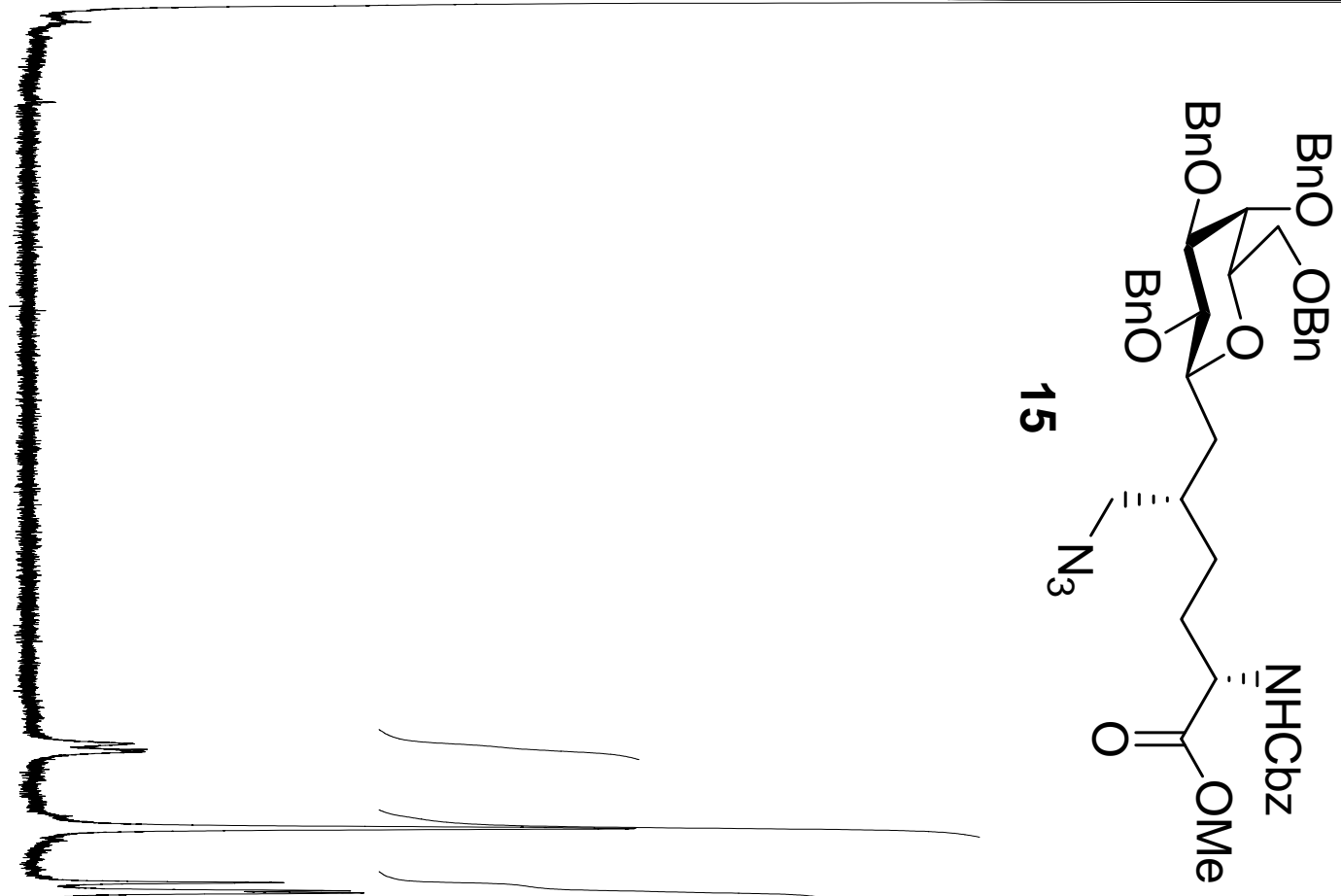

o

$98^{\circ} 0-\sqsubset$

0.
$0-\quad 66^{\circ}+$
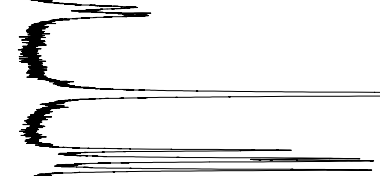

$\angle 0^{\circ}$

$68^{\circ} 2$

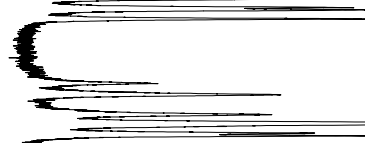

$-1 \begin{aligned} & 912- \\ & \text { 2म }\end{aligned}$

$+$

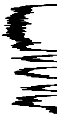

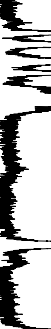

$99^{\circ} \mathrm{C}$

$0 t^{\circ}$

$\varepsilon 0$

90.

$00^{\circ} 2$

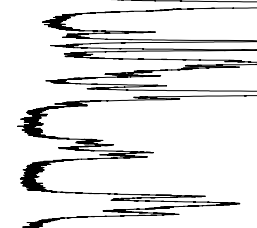

$\stackrel{\omega}{\circ}$

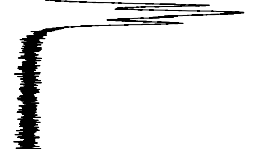

N

$\angle 0^{\circ} \varepsilon$

$0 Z^{\prime}$ เ

29

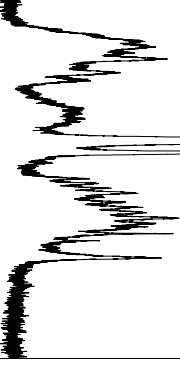




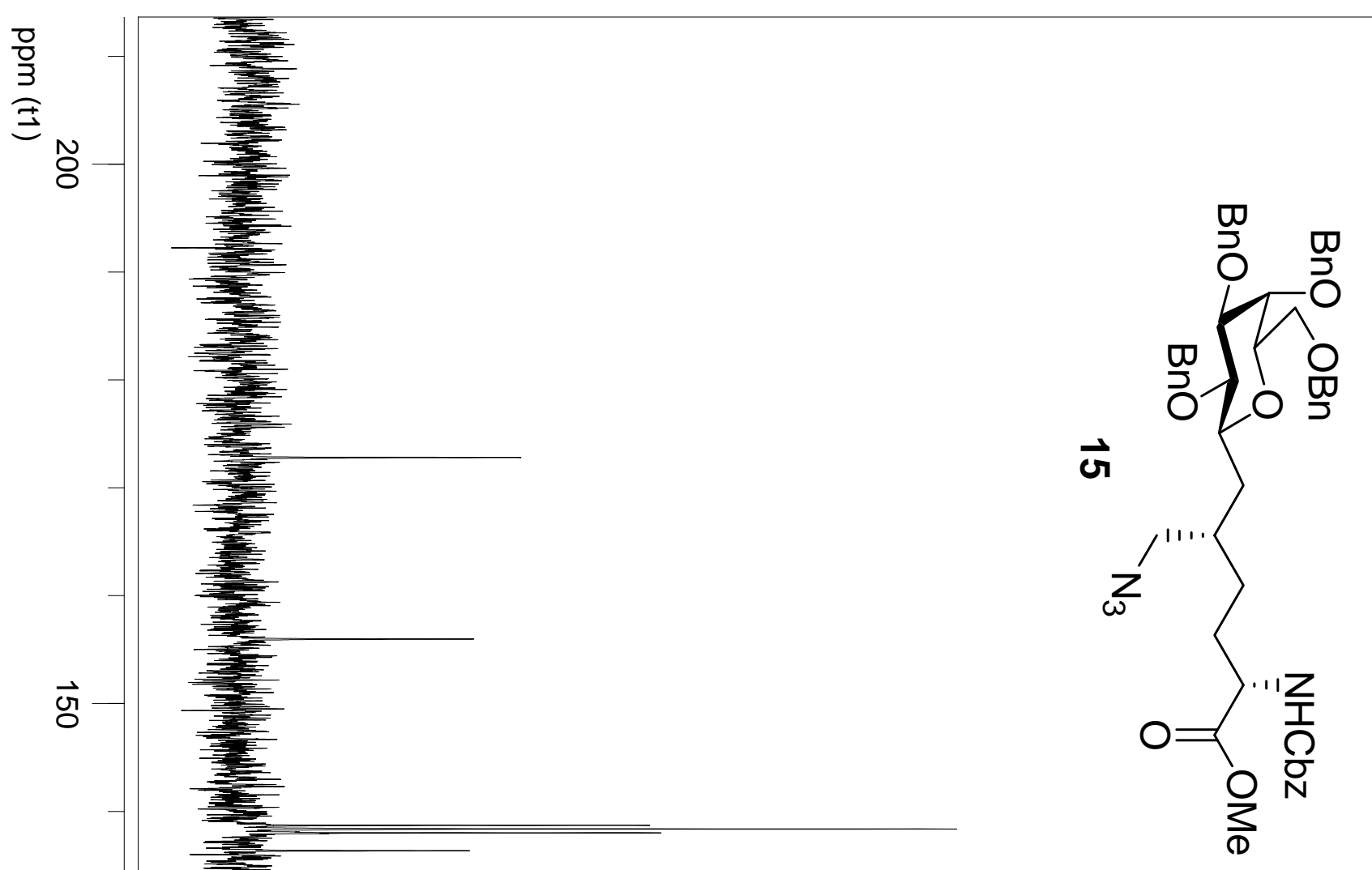

$\overrightarrow{8}$ 
$\frac{0}{3}$
3

$9 L 82$

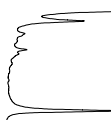

o

ह8.

$8 \varepsilon^{\prime \prime}$

80 '

0

SI'

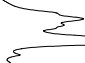

官

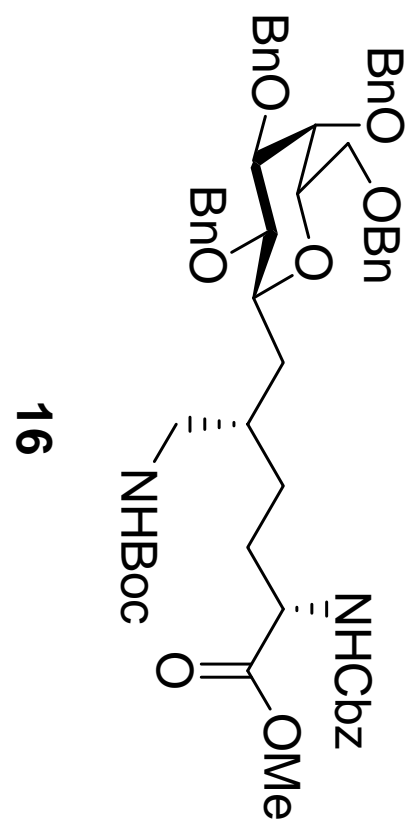

IG

81'

Gㄴ.

$26^{\circ} 0$

$\stackrel{\circ}{0}$

$\varepsilon 0^{\circ}$ เ

$0)^{\circ} \varepsilon$

$16^{\circ} \varepsilon$

$\angle 0^{\circ}$ '

ELเ

S० 2

$\omega$

$00^{\circ}$ เ

$00 \%$

N

$9 \nabla^{\circ} \varepsilon$

$18^{\circ} t$

$86^{\circ}$ เ 

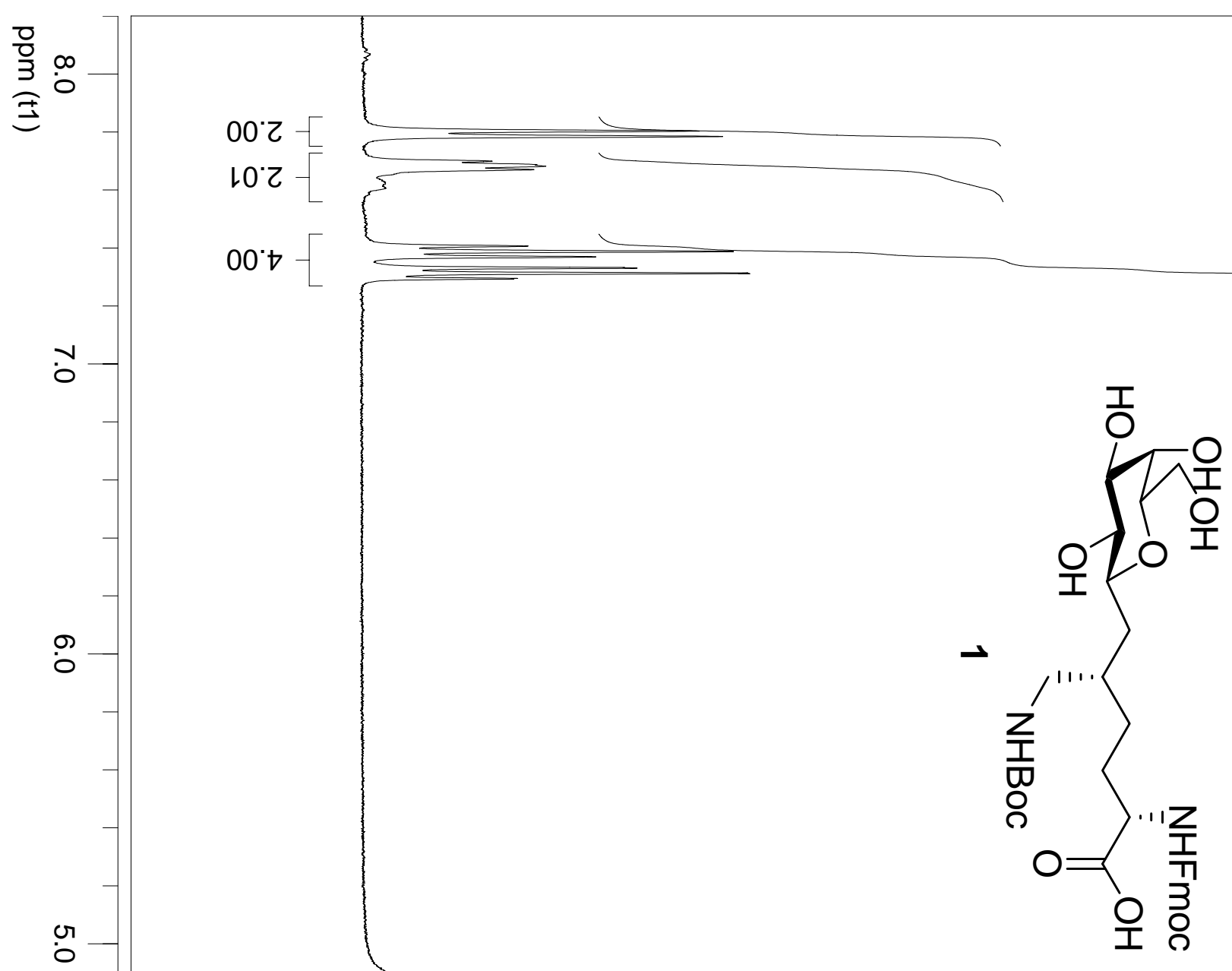

$\ddot{0}$

$60 \%$

$20 \%$

$68^{\circ} 0$

$50^{\circ}$

เレ

$67 * t$

$\omega$

OZ' -

LL'

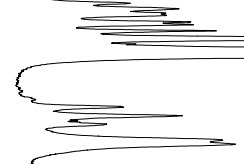

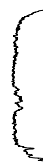

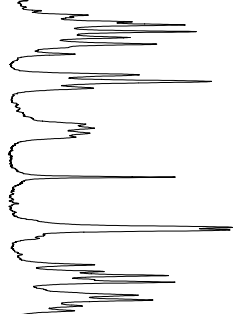

ह

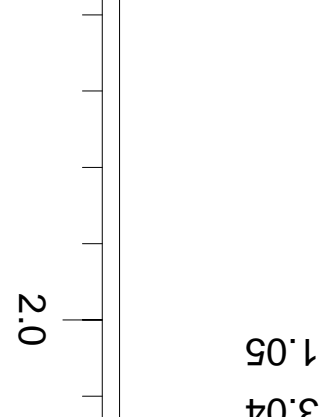

ยナ゙レ

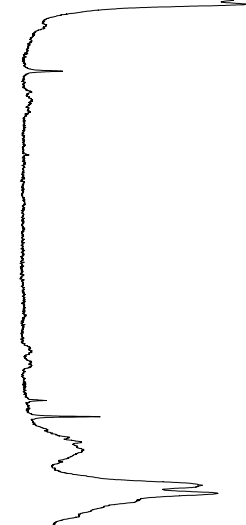




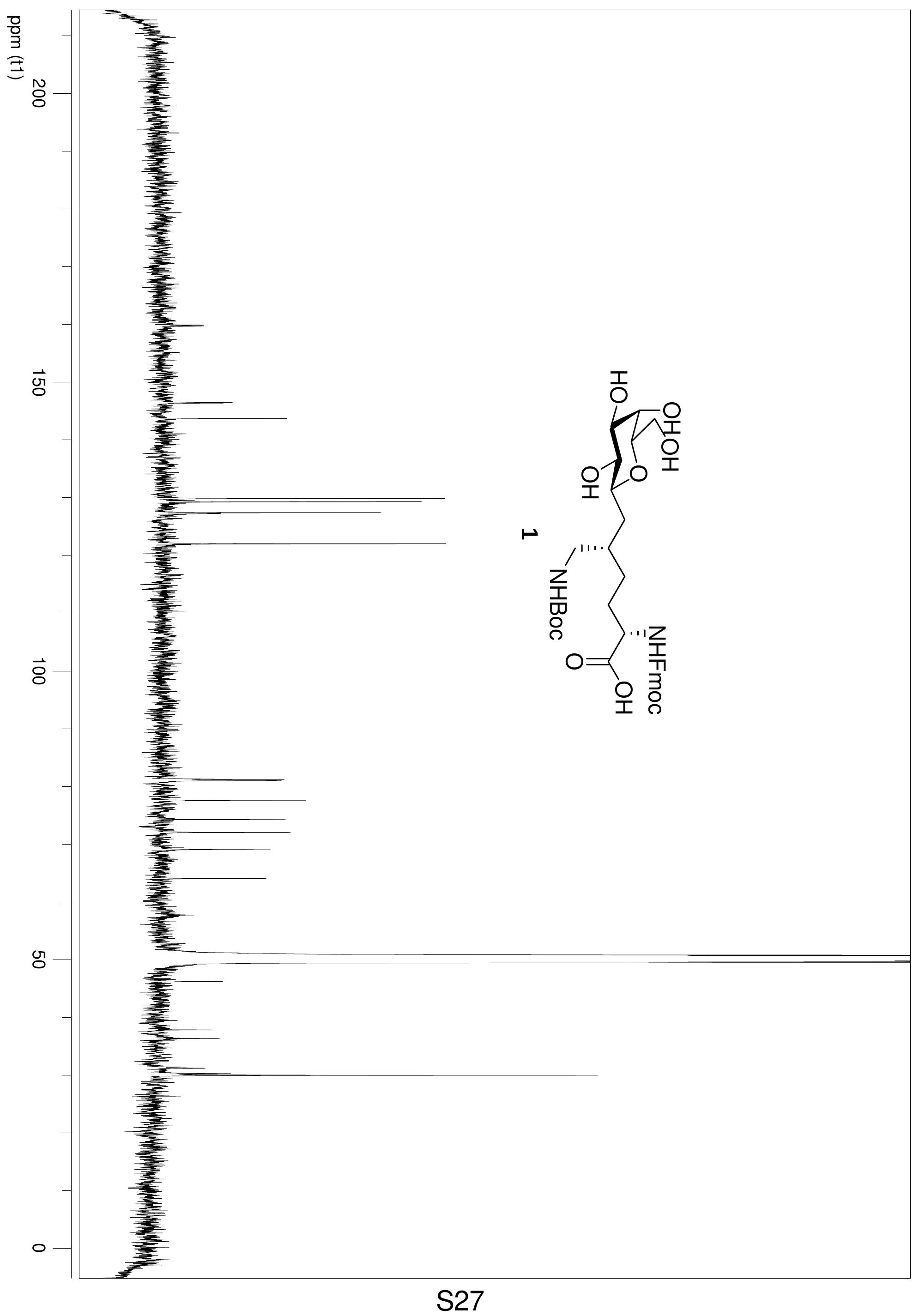

